\title{
Steady transcritical flow over an obstacle: Parametric map of solutions of the forced extended Korteweg-de Vries equation
}

\author{
Bernard K. Ee, ${ }^{1, a)}$ R. H. J. Grimshaw, ${ }^{2, b)}$ K. W. Chow, ${ }^{3, c)}$ and D-H. Zhang ${ }^{3, d)}$ \\ ${ }^{1}$ Department of Mathematics and Statistics, King Fahd University of Petroleum and Minerals, Dhahran \\ 31261, Saudi Arabia \\ ${ }^{2}$ Department of Mathematical Sciences, Loughborough University, Loughborough LE11 3TU, United \\ Kingdom \\ ${ }^{3}$ Department of Mechanical Engineering, University of Hong Kong, Pokfulam Road, Hong Kong
}

(Received 26 December 2010; accepted 2 April 2011; published online 21 April 2011)

\begin{abstract}
Transcritical flow of a stratified fluid over an obstacle is often modeled by the forced Korteweg-de Vries equation, which describes a balance among weak nonlinearity, weak dispersion, and small forcing effects. However, in some special circumstances, it is necessary to add an additional cubic nonlinear term, so that the relevant model is the forced extended Korteweg-de Vries equation. Here we seek steady solutions with constant, but different amplitudes upstream and downstream of the forcing region. Our main interest is in the case when the forcing has negative polarity, which represents a hole. The effects of the width of the hole and the amplitude of the hole on these steady solutions are then investigated. (C) 2011 American Institute of Physics. [doi:10.1063/1.3582523]
\end{abstract}

\section{INTRODUCTION}

The forced Korteweg-de Vries (fKdV) equation is often used as a suitable model to describe transcritical flow of a stratified fluid over an obstacle or through a contraction, see Grimshaw and Smyth ${ }^{1}$ and Clarke and Grimshaw, ${ }^{2}$ respectively. It is given by

$$
A_{t}+\Delta A_{x}+r A A_{x}+s A_{x x x}=-G_{0} f_{x},
$$

where $A(x, t)$ is the amplitude of the relevant linear long wave mode, $x, t$ are the space and time coordinates, respectively, and $\Delta$ is the linear long wave phase speed in the reference frame of the obstacle. The nonlinear and dispersive coefficients, $r, s$ respectively, are determined by the background stratification, $G_{0}$ is a measure of the forcing magnitude, and $f(x)$ is a projection of the obstacle (or contraction) onto the relevant long wave mode. A recent review of this model, including an outline of its derivation, has been given by Grimshaw. ${ }^{3}$

At the next order in a perturbation expansion in the amplitude, a higher-order Korteweg-de Vries equation is obtained, ${ }^{4}$ where cubic nonlinear, fifth-order linear dispersive, and nonlinear dispersive terms are added to Eq. (1). The contribution of these higher-order terms depends on the configuration of the model under investigation. A situation of special interest arises when the coefficient of the quadratic nonlinear term is close to zero. This situation arises, for instance, in a two-layer fluid in the Boussinesq approximation of nearly coincident densities, when the layer depths are nearly equal, see Baines. ${ }^{5}$ In this case a cubic nonlinear term is the dominant higher-order term, and a rescaling is needed,

\footnotetext{
${ }^{a)}$ Electronic mail: kwee@kfupm.edu.sa.

${ }^{b)}$ Electronic mail: r.h.j.grimshaw@lboro.ac.uk.

${ }^{c}$ Electronic mail: kwchow@hku.hk.

${ }^{d)}$ Electronic mail: dzhang @hkucc.hku.hk.
}

after which forced Korteweg-de Vries equation (1) is replaced by the forced extended $\mathrm{KdV}(\mathrm{feKdV})$ equation, also known as the forced Gardner equation,

$$
A_{t}+\Delta A_{x}+r A A_{x}+q A^{2} A_{x}+s A_{x x x}=-\gamma f_{x} .
$$

In comparison with $\mathrm{fKdV}$ equation (1), there is now an additional cubic nonlinear term with coefficient $q$. Consequent to the rescaling, we can assume that the coefficients of the quadratic and cubic nonlinear terms have the same order of magnitude.

Importantly, there are two distinct equations depending on the relative polarity between the cubic nonlinear term and the dispersive term. ${ }^{6-8}$ There appear to be very few studies of eKdV equation (2), although we note two relevant exceptions here. Choi et al. ${ }^{9}$ derived Eq. (2) for capillary-gravity waves in a two-layer fluid generated by moving a small localized obstruction. They described solutions for both cases $q s<0$ and $q s>0$ but considered only solitary waves or periodic waves and did not discuss any hydraulic solutions. Grimshaw et al. ${ }^{10}$ employed the long-wave approximation for localized forcing to find hydraulic solutions for various parametric configurations of the feKdV equation when $q s$ $<0$ and derived a criterion for which steady hydraulic solutions could form.

Transcritical flow over a step has been investigated in the framework of fKdV equation (1) (Ref. 11) and compared with simulations of the full Euler equations. ${ }^{12}$ In essence, asymptotic solutions consisting of steady, hydraulic solutions in the vicinity of an isolated step were constructed and matched with upstream and downstream undular bores as appropriate. These works demonstrate that positive and negative steps generated upstream and downstream propagating undular bores, respectively. The general definition of the polarity the forcing term is made below, but we note that for water waves it corresponds to a hill or a hole, respectively. In the case of a long hole ${ }^{13}$ (comprising a negative step fol- 
lowed by a positive step), downstream and upstream undular bores were generated and found to interact over the hole.

In this paper we extend the study by Ee et al. ${ }^{14}$ which examined the steady hydraulic solutions of fKdV equation (1). They determined the parametric relationship $\Delta=\Delta(\gamma)$ for the existence of steady hydraulic solutions. Loops in this parametric map indicated the number of trapped waves found over a hole, while no such trapped waves were found over a hill. Our main aim here is to determine the analogous parametric relationship $\Delta=\Delta(\gamma)$ for the existence of steady hydraulic solutions for forced $\mathrm{KdV}$ equation $\backslash(2)$. We expect that with two nonlinear terms, the parametric relationships and the types of steady solutions that can be formed will be more intricate.

The numerical procedure used in Ee and Clarke ${ }^{15}$ will again be employed in this paper. Briefly, a shooting algorithm was implemented alongside a minimization algorithm in order to obtain the steady dispersive hydraulic solution to Eq. (1) over a localized forcing term, which may have a long length scale. Then a branch-following algorithm is developed to obtain the parametric relationship describing the family of steady hydraulic solutions. In our numerical study, we choose the forcing term to be

$$
f(x)=\frac{1}{2} \tanh \left(\xi\left[x-\frac{L}{2}\right]\right)-\frac{1}{2} \tanh \left(\xi\left[x+\frac{L}{2}\right]\right),
$$

where $L$ is the separation between the front and rear steps and $1 / \xi$ measures the width of these steps. Forcing term (3) is symmetric and centered at $x=0$, where $x<-L / 2$ and $x>L / 2$ denote the upstream and downstream regions, respectively.

In Sec. II, we shall present the problem in canonical form to identify the relevant parameters. In Sec. III we shall consider the case of a piecewise constant forcing when an analytical solution can be found using phase plane analysis. Then in Sec. IV we use a slowly varying approximation to supplement the results from the case of piecewise constant forcing. In Sec. V, a brief outline of the numerical method is followed by a discussion of our numerical results for smooth forcing term (3). Finally, we conclude with a summary of our key results in Sec. VI.

\section{FORMULATION}

First we transform Eq. (2) to a canonical form in order to reduce the parameter space to just four parameters. Let $\tilde{x}$ $=\xi x, \tilde{\tau}=s \xi^{3} t, \tilde{L}=\xi L$, and $A(x, t)=A_{0}+B(x, t)$, where $A_{0}$ is a characteristic upstream amplitude. Then $B(x, t)$ satisfies the feKdV equation,

$$
B_{t}+\Delta B_{x}+6 B B_{x}+3 q B^{2} B_{x}+B_{x x x}=-\gamma f_{x},
$$

where

$$
\begin{aligned}
& \frac{r+2 A_{0}}{s \xi^{2}}=6, \quad \widetilde{\Delta}=\frac{6\left(\Delta+r A_{0}+q A_{0}^{2}\right)}{r+2 A_{0}}, \\
& 3 \widetilde{q}=\frac{6 q}{r+2 A_{0}}, \quad \tilde{\gamma}=\frac{6 \gamma}{r+2 A_{0}},
\end{aligned}
$$

and the tildes have been omitted from Eq. (4). With this rescaling, forcing term (3) then becomes

$$
f(x)=\frac{1}{2} \tanh \left(x-\frac{L}{2}\right)-\frac{1}{2} \tanh \left(x+\frac{L}{2}\right),
$$

where $L$ is the fourth parameter (again the tilde has been omitted) which we shall vary independently in order to investigate its effect on the parametric map. Note that -1 $\leq f(x) \leq 0$, and there is a minimum at $x=0$, where $f(0)=-1$ and $f \rightarrow 0$ as $|x| \rightarrow \infty$. We define negative or positive forcing accordingly as $\gamma>0$ or $\gamma<0$, respectively. The case $\gamma>0$ is our main concern in this paper, and we shall call this case a hole.

As stated in Sec. I, our concern here is with the steady dispersive hydraulic solutions. Hence, we set the time derivative term in Eq. (4) to zero. One integration then yields

$$
\Delta B+3 B^{2}+q B^{3}+B_{x x}=-\gamma f(x),
$$

where the boundary condition that $B \rightarrow 0, x \rightarrow-\infty$ has been imposed. In order to obtain some insight into the numerical solutions we shall describe in Sec. V, we shall suppose in this section that forcing term (6) is a compact forcing term where $f(x)=0, x \leq-L / 2, x \geq L / 2$, and $f(x)$ varies smoothly and monotonically from 0 at $x=-L / 2$ to a minimum of -1 at $x$ $=0$, and then returns monotonically to 0 at $x=L / 2$. Positive (negative) forcing corresponds to $\gamma<(>) 0$. In Sec. III which follows we shall explore this situation in more detail when $f(x)=-1,-L / 2<x<L / 2$. To proceed, we must first determine the critical points of the unforced equation, given by

$$
B_{0}=0, \quad B_{1,2}=\frac{-3 \pm \sqrt{9-4 \Delta q}}{2 q} .
$$

First suppose that $\Delta>0$, in which case $B_{0}=0$ is a center. It follows that no solitary waves are allowed and, upstream, we can set

$$
\begin{aligned}
& B=0 \text { for } x<-L / 2, \\
& \text { so that } B(x=-L / 2)=0, \quad B_{x}(x=-L / 2)=0 .
\end{aligned}
$$

Downstream, there are two cases to consider, (1) $q<0$ and (2) $q>0$. In case (1) $B_{1}<0<B_{2}$ and both $B_{1,2}$ are saddle points. A typical phase plane is shown in Fig. 1. The main interest here are the orbits through the critical point $B_{1}$ (solid curves); note that the saddle point at $B=B_{2}$ is not shown. This homoclinic orbit is the solitary wave,

$$
\begin{aligned}
& B=B_{1}+\frac{a}{1+\beta \cosh [\kappa(x-L / 2)+\theta]}, \quad x>L / 2, \\
& \kappa^{2}=-\Delta-6 B_{1}-3 q B_{1}^{2}=a\left(1+q B_{1}\right),
\end{aligned}
$$




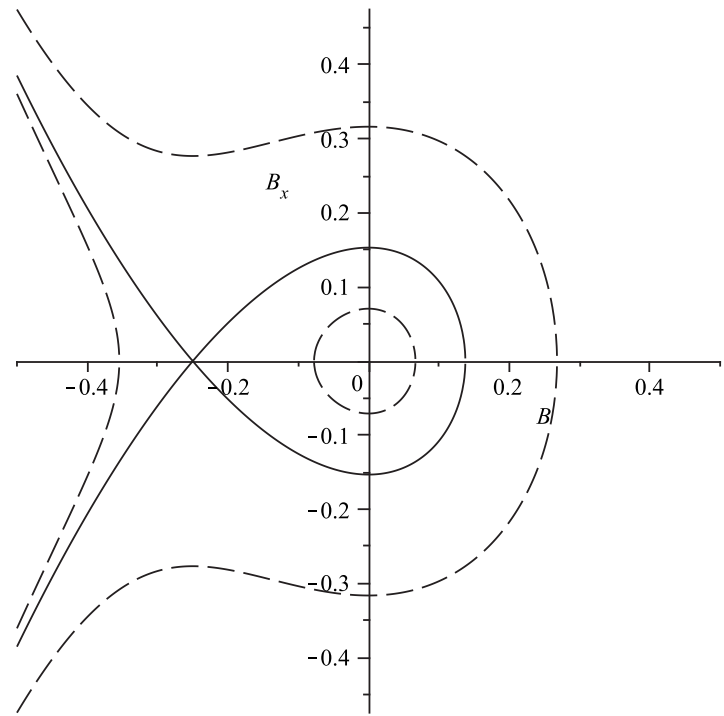

FIG. 1. Phase plane of unforced Eq. (7) for $\Delta=1, q=-4$.

$$
\kappa^{2}\left(1-\beta^{2}\right)=-\frac{q a^{2}}{2} .
$$

Here $\theta$ is a phase constant to be determined by matching with the solution in the forcing region. Note that with $q<0$, it follows that $0<\beta<1, a>0$, and the constraint that $\Delta$ $>0$ implies that $a\left(1+q B_{1}\right)<-B_{1}\left(6+3 q B_{1}\right)$. The amplitude $a_{s}=a /(1+\beta)=2\left\{-1-q B_{1}+\left(1+q B_{1} / 2\right)^{1 / 2}\right\} / q$ and is such that $0<B_{1}+a_{s}<B_{2}$. The unbounded orbits (outer dash curves) are given by

$B=B_{1}+\frac{a}{1+\beta \sinh [\kappa(x-L / 2)+\theta]}, \quad x>L / 2$,

$\kappa^{2}=-\Delta-6 B_{1}-3 q B_{1}^{2}=a\left(1+q B_{1}\right), \quad \kappa^{2}\left(1+\beta^{2}\right)=-\frac{q a^{2}}{2}$.

Formally, this is obtained from Eq. (10) by replacing $\theta$ with $\theta+i \pi / 2$ and $\beta$ with $-i \beta$. If needed, the analogous singular orbits through $B_{2}$ are found from Eq. (11) by replacing $B_{1}$ with $B_{2}$.

In case (2) there are two subcases, (a) when $4 \Delta q<9$ and (b) when $4 \Delta q>9$. In the first case (2a) $B_{2}<B_{1}<0$ are a center and a saddle point, respectively. A typical phase plane is shown in Fig. 2. Here again the main interest is the homoclinic orbit (solid curve) through the critical point $B=B_{1}$ which is again given by Eq. (10). Now $\beta^{2}>1$, and the branch around the center at $B=0$ has amplitude $a_{s 1}=a /$ ( 1 $+\beta)=2\left\{-1-q B_{1}+\left(1+q B_{1} / 2\right)^{1 / 2}\right\} / q>0$, while the branch around the center at $B_{2}$ has amplitude $a_{s 2}=a /(1+\beta)=2\{-1$ $\left.-q B_{1}-\left(1+q B_{1} / 2\right)^{1 / 2}\right\} / q<0$. It can be shown that $B_{1}+a_{s 2}$ $<B_{2}<B_{1}<0<B_{1}+a_{s 1}$. If $1+q B_{1}>0, \Delta q<2, a>0$, then the branch around the center at $B=0$ has $1<\beta<\infty$, while the branch around the center at $B_{2}$ has $-\infty<\beta<-1$. But if 0 $>1+q B_{1}>-1 / 2,2<\Delta q<9 / 4, a<0$, then these branches interchange. Note that the constraint $\Delta>0$ implies that $a(1$ $\left.+q B_{1}\right)<-B_{1}\left(6+3 q B_{1}\right)$. In the second case $(2 \mathrm{~b})$, there is only one critical point, the center at $B=0$, and all orbits are periodic.

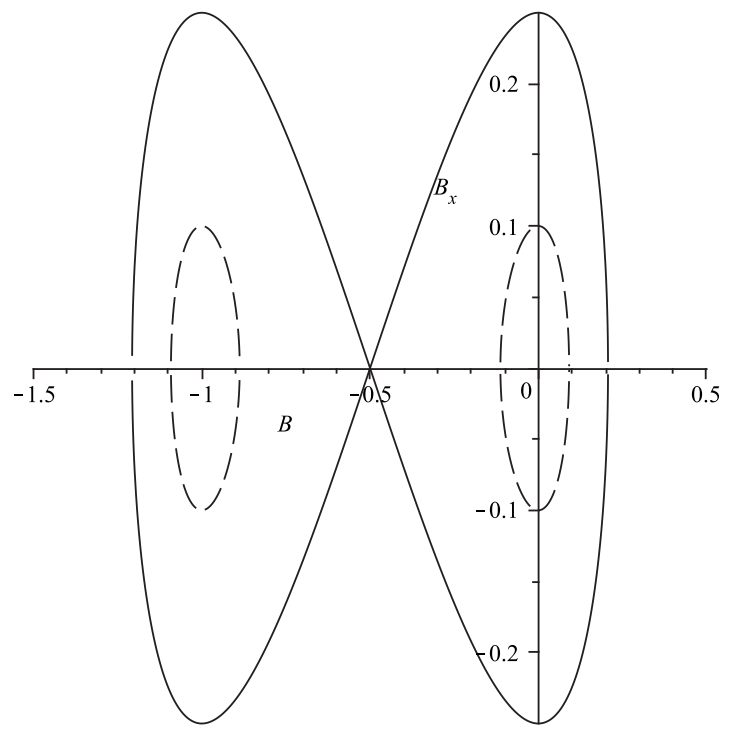

FIG. 2. Phase plane of unforced Eq. (7) for $\Delta=1, q=2$

We can now specify the downstream boundary conditions, where we seek a solution which tends to an available saddle point as $x \rightarrow \infty$. As we show below, this leads to a third boundary condition in addition to the two upstream boundary conditions (9), and hence solutions, in general, exist only when there is a parametric relation $\Delta=\Delta(\gamma)$. For case (1) $(q<0)$ and case $(2 \mathrm{a})(q>0,4 \Delta q<9)$, we first assume that the solution tends to the critical point $B_{1}$ through solitary wave solution (10), so that

$$
\begin{aligned}
& B(x=L / 2)=B_{1}+\frac{a}{1+\beta \cosh \theta}, \\
& B_{x}(x=L / 2)=-\frac{a \kappa \beta \sinh \theta}{(1+\beta \cosh \theta)^{2}} .
\end{aligned}
$$

Elimination of $\theta$ leads to a single nonlinear boundary condition to determine the solitary wave parameter $\beta$. If instead the solution tends to the critical point $B_{1}$ through unbounded orbit (11), then boundary condition (12) is replaced by

$$
\begin{aligned}
& B(x=L / 2)=B_{1}+\frac{a}{1+\beta \sinh \theta}, \\
& B_{x}(x=L / 2)=-\frac{a \kappa \beta \cosh \theta}{(1+\beta \sinh \theta)^{2}} .
\end{aligned}
$$

In case (2b) $(q>0,4 \Delta q>9)$, there is no available homoclinic orbit, and we infer that, in general, there is no solution. Next we note that in case $(1)(q<0)$ there is the possibility that the solution tends to the critical point $B_{2}$ through an unbounded orbit, in which case boundary condition (13) is modified by replacing $B_{1}$ with $B_{2}$. There remains the possibility that the downstream solution tends to a center, either $B=0$ in all cases or $B_{2}$ in case (2a), leading to the downstream boundary conditions, 


$$
B(x=L / 2)=0 \text { or } B_{2}, \quad B_{x}(x=L / 2)=0 .
$$

But when combined with upstream boundary condition (9), this leads to four boundary conditions, and hence we infer that such solutions exist only in very exceptional circumstances.

Next suppose that $\Delta<0$, in which case $B_{0}$ is a saddle point. A solitary wave now exists upstream, given by [compare Eq. (10) with $\left.B_{1}=0\right]$

$$
\begin{aligned}
& B=\frac{a}{1+\beta \cosh (\kappa(x+L / 2)+\phi)}, \quad x<-L / 2, \\
& \kappa^{2}=-\Delta=a, \quad \kappa^{2}\left(1-\beta^{2}\right)=-\frac{q a^{2}}{2} .
\end{aligned}
$$

Note that $a>0$ and $\beta^{2}>1,<1$ accordingly as $q<0,>0$. The upstream boundary condition is then

$$
\begin{aligned}
& B(x=-L / 2)=\frac{a}{1+\beta \cosh \phi}, \\
& B_{x}(x=-L / 2)=-\frac{a \kappa \beta \sinh \phi}{(1+\beta \cosh \phi)^{2}} .
\end{aligned}
$$

Downstream, there are again two cases to consider, (1) $q$ $<0$ and (2) $q>0$. In case (1) there are now two subcases, (a) when $4 \Delta q<9$ and (b) when $4 \Delta q>9$. In the first case (1a), $0<B_{1}<B_{2}$ are a center and a saddle point, respectively, while in the second case there is only one critical point, the saddle point $B=0$. In case (2) $B_{2}<0<B_{1}$ and are both centers. If we now suppose that downstream the solution tends to a center, say $B_{c}$, then we can change variables $\widetilde{B}=B$ $-B_{c}, \widetilde{x}=-x$, and with a suitable rescaling, we recover the case when $\Delta>0$ discussed above.

Hence the new feature here is the possibility that downstream the solution tends to a saddle point, either $B=0$ in all cases or $B_{2}$ in case (1a). For the former case, the solitary wave is given by Eq. (10), but with $B_{1}$ replaced by 0 , so that the downstream boundary condition is

$$
\begin{aligned}
& B(x=L / 2)=\frac{a}{1+\beta \cosh \theta}, \\
& B_{x}(x=L / 2)=-\frac{a \kappa \beta \sinh \theta}{(1+\beta \cosh \theta)^{2}} .
\end{aligned}
$$

For the latter case, the solitary wave is given by Eq. (10), but with $B_{1}$ replaced by $B_{2}$, and the downstream boundary condition is then adapted from Eq. (12) accordingly. We see that only two boundary conditions required and hence solutions can be found without the necessity to seek a parametric relation between $\Delta$ and $\gamma$. To illustrate this situation, let us choose the forcing function as

$$
f(x)=-\frac{(1+\beta)^{n}}{(1+\beta \cosh \kappa x)^{n}}, \quad n=1,2,3 .
$$

Note that this is not compact, but is localized as $f(x) \rightarrow 0, x$ $\rightarrow \pm \infty$. We seek a solution in the form of a solitary wave,

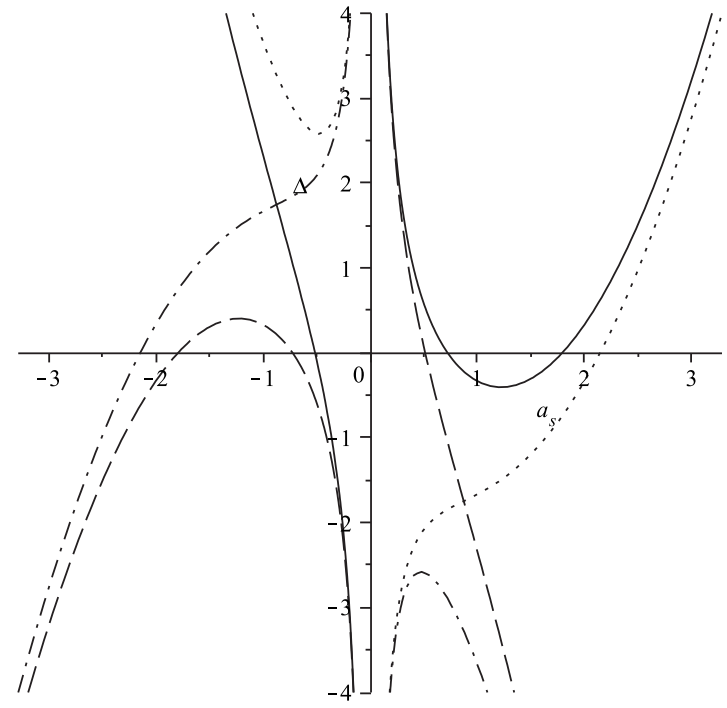

FIG. 3. Plot of bifurcation curve (21) for $n=2$, where only the portions $\Delta$ $<0$ are legitimate. The cases $(\gamma, q)=(1,-1),(1,1),(-1,-1),(-1,1)$ correspond to the solid, dash, dot, and dash-dot curves, respectively.

$$
B(x)=\frac{a_{s}(1+\beta)}{1+\beta \cosh \kappa x} .
$$

Then we get that, for $n=1,2,3$, respectively,

$$
\begin{aligned}
& \frac{1-\beta}{1+\beta} 2 \kappa^{2} a_{s}+q a_{s}^{3}=0,0, \gamma, \\
& -\frac{3 \kappa^{2} a_{s}}{1+\beta}+3 a_{s}^{2}=0, \gamma, 0, \\
& \kappa^{2} a_{s}+\Delta a_{s}=\gamma, 0,0 .
\end{aligned}
$$

In each case, there are three equations for the three parameters $a, \beta, \kappa$, and hence, in general, a solution can be found as functions of $\Delta$ and $\gamma$. Note that for the cases $n=2,3$, when the forcing term vanishes at infinity faster than the solution, a desirable constraint, then we must have $\Delta<0$. In each case, elimination of $\kappa^{2}, \beta$ yields a bifurcation curve $\Delta=\Delta\left(a_{s}\right)$ for each fixed value of $\gamma$, given by

$$
\begin{aligned}
& \Delta a_{s}+2 a_{s}^{2}-\frac{q}{2} a_{s}^{3}=\gamma, \quad n=1, \\
& \frac{3 \Delta}{2} a_{s}+3 a_{s}^{2}+\frac{3 q}{4} a_{s}^{3}=\gamma, \quad n=2, \\
& -2 \Delta a_{s}+4 a_{s}^{2}+q a_{s}^{3}=\gamma, \quad n=3 .
\end{aligned}
$$

With suitable changes of variable, these are qualitatively similar curves. Also in all cases, the transposition $a_{s} \rightarrow-a_{s}, q \rightarrow-q, \Delta \rightarrow-\Delta$ leaves each curve unchanged. The case $n=2$ is plotted in Fig. 3. On these curves, the parameter is $\kappa^{2}$, which implies that for $n=2,3$, only the portions where $\Delta<0$ are legitimate, while for $n=1$, there is the constraint that $a_{s}\left(4-q a_{s}\right)>0$. All the curves can be interpreted as perturbations from the unforced solutions when $\gamma=0$, which are either the uniform stream when $a_{s}=0$ or solitary wave (15). 


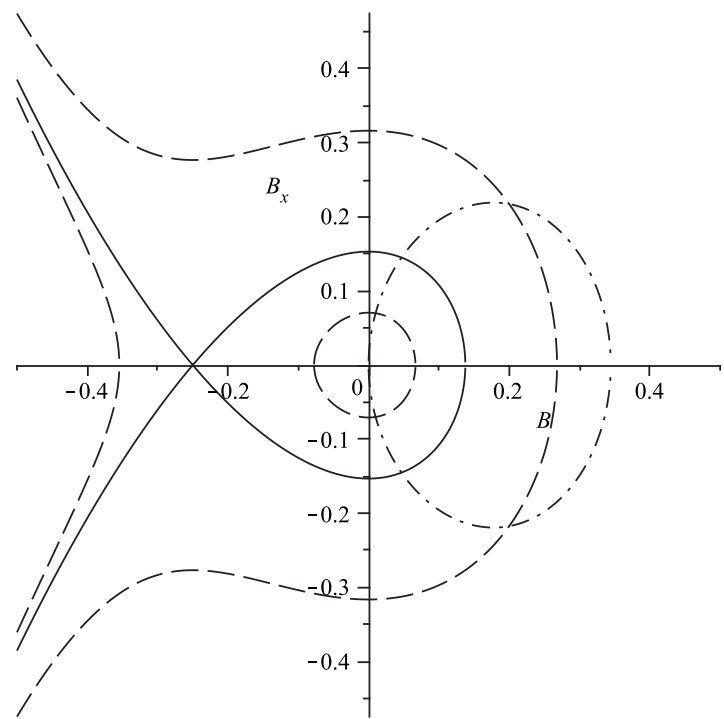

FIG. 4. Phase plane for unforced Eq. (7) with $\Delta=1, q=-4$, showing a periodic orbit for the forced equation when $\gamma=0.25$ (dash-dot curve).

At the turning points where $d \Delta / d a_{s}=0$, we expect a change of stability on each curve. Since a uniform stream is stable within the framework of feKdV equation (2), we can infer that the curves which represent a perturbation from a uniform stream are stable until a turning point is reached, beyond which they represent unstable solutions. This is essentially the same result described by Grimshaw et al. ${ }^{16}$ for the stability of localized steady solutions of the $\mathrm{fKdV}$ equation. Note that $q=0$ recovers the forced $\mathrm{KdV}$ case, when $n=1,2$ are well-known solutions, but the case $n=3$ is apparently a previously unnoticed solution.

\section{PIECEWISE CONSTANT FORCING}

We now let $f=-1$ in $-L / 2<x<L / 2$, so that the forcing is a nonzero constant in the forcing region. The solution in the forcing region can now be found explicitly using a phase plane analysis, and then matched with the solutions outside the forcing region using the continuity of $B, B_{x}$ at $x=$ $-L / 2, L / 2$. This phase plane approach was used by Dias and VandenBroeck, ${ }^{17-19}$ Binder et al., ${ }^{20,21}$ and Grimshaw et al. ${ }^{13}$ for fKdV equation (1), and by Dias and Vanden-Broeck ${ }^{22}$ for forced extended $\mathrm{KdV}$ equation (2), for a variety of transcritical flows over steps and obstacles.

\section{A. Negative forcing: $\gamma>0$}

First take $\Delta>0$ and $q<0$, case (1). In the forcing region, there are either three critical points $C_{1}<C_{0}<C_{2}$, respectively, saddle point, center, saddle point being the counterpart of Eq. (8) when $0<\gamma<\gamma_{c}$, or just one critical point $C_{1}<0$ when $\gamma>\gamma_{c}$ where $q^{2} \gamma_{c}=2(1-q \Delta / 3)^{3 / 2}+2-q \Delta$. Relative to critical points (8), $C_{1}<B_{1}<0<C_{0}<C_{2}<B_{2}$. A typical scenario is shown in Fig. 4 which is the same case as Fig. 1, now extended to include the relevant orbit from the phase plane for $-L / 2<x<L / 2$. The direction of increasing $x$ is clockwise. The task is to connect the origin of the phase plane with the homoclinic orbit (solid curve) through the saddle points $B=B_{1}$ or the unbounded orbit (not shown)

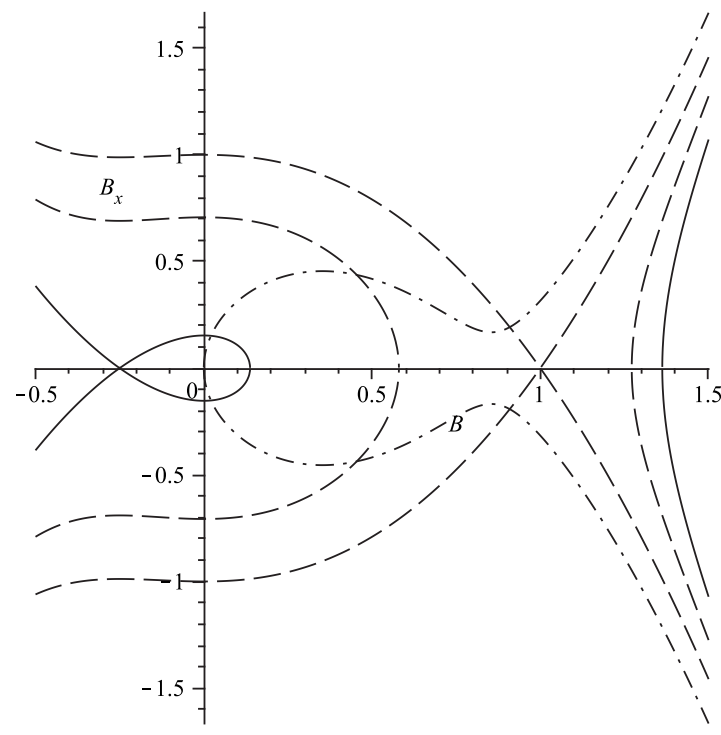

FIG. 5. Phase plane for unforced Eq. (7) with $\Delta=1, q=-4$, showing an unbounded orbit for the forced equation when $\gamma=0.55$ (dash-dot curve).

through $B_{2}$, using the relevant orbit (dash-dot curve) from the forcing region $-L / 2<x<L / 2$. Figure 4 shows a case when the orbit from the forcing region phase plane is a periodic orbit intersecting the homoclinic orbit at $x=X$, say. However, in general, $X \neq L / 2$, and so the values of $\Delta, \gamma$ must be then adjusted until $X=L / 2$. It may be necessary to go around the periodic orbit several times, corresponding to several wave crests. Also there are two possible cases; one where the intersection with the homoclinic orbit is for $B_{x}<0, \theta>0$, and so the solitary wave in $x>L / 2$ is suppressed; the other has $B_{x}>0, \theta<0$ and so there is a partial solitary wave in $x$ $>L / 2$. Note that since $C_{0}<C_{2}<B_{2}$, there can be no intersections of the periodic orbit with the unbounded orbit through $B_{2}$. Decreasing $\gamma$, such that $0<\gamma<\gamma_{c_{1}}$, leads to no intersection, where $\gamma_{c 1}$ is determined by the criterion that the periodic orbit through the origin should lie inside the homoclinic orbit defined by Eq. (10), that is, $2 \gamma_{c 1}=\Delta B_{m}^{2}+2 B_{m}^{3}$ $+q B_{m}^{4} / 2$, where $B_{m}$ is the amplitude of the homoclinic orbit, that is, $B_{m}=B_{1}+a_{s}$. But increasing $\gamma$ so that $\gamma>\gamma_{c 2}$, where $q^{2} \gamma_{c 2}=32 / 27\left\{1-9 \Delta q / 16+(1-3 \Delta q / 8)^{3 / 2}\right\}$, leads to the orbit in the forcing region through the origin of the phase plane being an unbounded orbit associated with the saddle point at $C_{2}$ for $\gamma_{c 2}<\gamma<\gamma_{c}$, and then with an unbounded orbit associated with the saddle point at $C_{1}$ for $\gamma>\gamma_{c}$. This intersects both the homoclinic orbit through $B_{1}$ and the unbounded orbit through $B_{2}$. An example is shown in Fig. 5, which is the same case as Fig. 1, but now showing the unbounded orbits (dash curves) through the saddle point $B_{2}$. In this case there are no waves generated in the forcing region but connections can be made with $B_{1}$ through the homoclinic orbit (solid curve) and with $B_{2}$ through the unbounded orbit (dash curve). Note that $\gamma_{c 1}<\gamma_{c 2}<\gamma_{c}$.

Next take $\Delta>0$ and $q>0$ with $4 \Delta q<9$, case (2a). In the forcing region, there are either three critical points $C_{2}<C_{1}$ $<C_{0}$, respectively, center, saddle point, center being the counterpart of Eq. (8) when $0<\gamma<\gamma_{c}<0$, or just one critical point $C_{0}>0$ when $\gamma>\gamma_{c}$. Relative to critical points (8) 


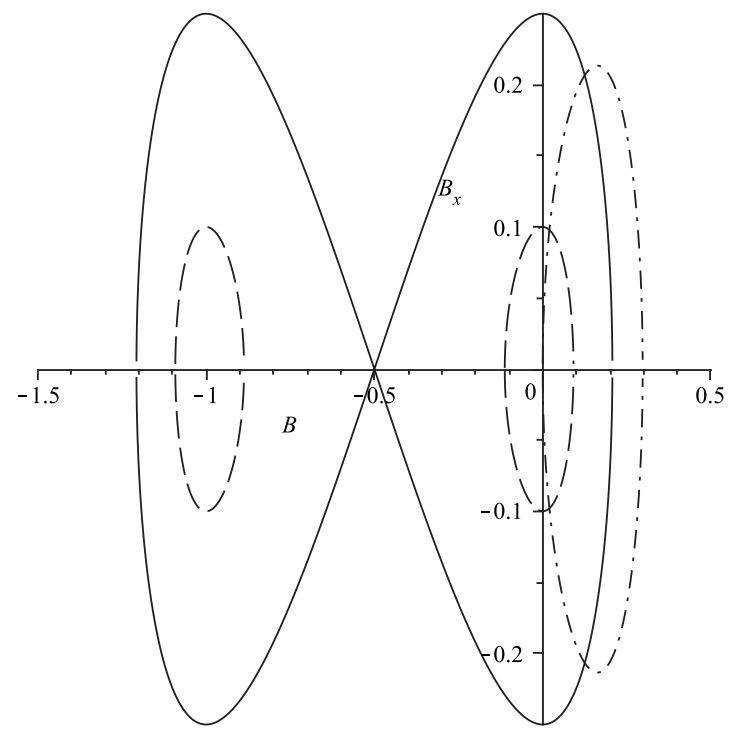

FIG. 6. Phase plane of unforced Eq. (7) for $\Delta=1, q=2$, showing a periodic orbit for the forced equation when $\gamma=0.25$.

$B_{2}<C_{2}<C_{1}<B_{1}<0<C_{0}$. A typical phase plane is shown in Fig. 6, which is the same case as Fig. 1, now extended to include the relevant orbit from the phase plane for $-L / 2$ $<x<L / 2$. Figure 6 shows a case when there is an intersection, which here is always a periodic orbit (dash-dot curve) in the forcing region intersecting the homoclinic orbit through $B_{1}$. Decreasing $\gamma$, so that $0<\gamma<\gamma_{c 1}<\gamma_{c}$, leads to no intersections at all.

\section{B. Positive forcing: $\gamma<0$}

The phase plane analysis can again be applied, but there are now major differences. First, take $\Delta>0$ and $q<0$, case (1). In the forcing region, there are either three critical points $C_{1}<C_{0}<C_{2}$, respectively, saddle point, center, saddle point when $\gamma_{c}<\gamma<0$, or just one critical point $C_{2}>0$ when $\gamma$ $<\gamma_{c}$ where now $q^{2} \gamma_{c}=-2(1-q \Delta / 3)^{3 / 2}+2-q \Delta$. Relative to critical points (8), $B_{1}<C_{1}<C_{0}<0<B_{2}<C_{2}$. In this case if the orbit through the origin of the phase plane in the forcing region is a periodic orbit around $C_{0}$, then this lies inside the homoclinic orbit through $C_{1}$ and inside the homoclinic orbit through $B_{1}$; hence there can be no intercepts. This situation arises whenever $\gamma_{c}<\gamma_{c 3}<\gamma<0$, where $q^{2} \gamma_{c 3}=32 / 27\{1$ $\left.-9 \Delta q / 16-(1-3 \Delta q / 8)^{3 / 2}\right\}$. A typical phase plane is shown in Fig. 7 when $\gamma_{c}<\gamma<\gamma_{c 3}$, which is the same case as Fig. 1, now extended to include the relevant orbit from the phase plane for $-L / 2<x<L / 2$. We see that there is only a single intercept between an unbounded orbit from the origin of the phase plane in the forcing region, with the homoclinic orbit through $B_{1}$ and this combined orbit has $B<0, B_{x}<0$. The case when $\gamma<\gamma_{c}$ is qualitatively the same.

Next take $\Delta>0$ and $q>0$ with $4 \Delta q<9$, case (2a). In the forcing region, there are either three critical points $C_{2}<C_{1}$ $<C_{0}$, respectively, center, saddle point, center being the counterpart of Eq. (8) when $\gamma_{c}<\gamma<0$, or just one critical point $C_{2}<0$ when $\gamma<\gamma_{c}$. Relative to critical points (8) $C_{2}$ $<B_{2}<B_{1}<C_{1}<C_{0}<0$. As above in the case $q<0$, if the orbit through the origin of the phase plane in the forcing

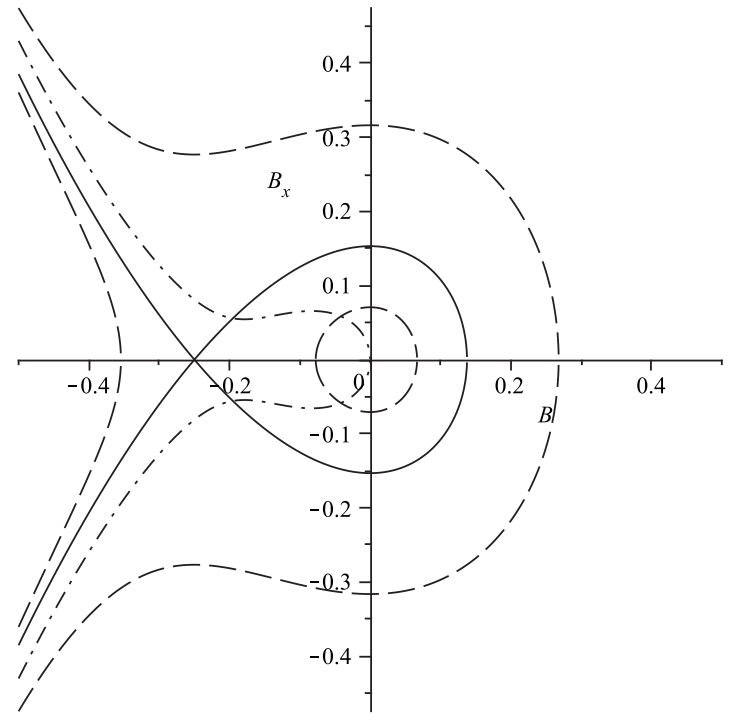

FIG. 7. Phase plane of unforced Eq. (7) for $\Delta=1, q=-4$, showing an unbounded orbit (dash-dot curve) for the forced equation when $\gamma=-0.06$.

region is a periodic orbit around $C_{0}$, then this lies inside the homoclinic orbit through $C_{1}$ and inside the homoclinic orbit through $B_{1}$; hence there can be no intercepts. This situation again arises whenever $\gamma_{c}<\gamma_{c 3}<\gamma<0$, where $\gamma_{c}, \gamma_{c 3}$ are defined as in the previous paragraph. However, for $\gamma_{c}<\gamma$ $<\gamma_{c 3}$ when the homoclinic orbit through $C_{1}$ contains the origin, there is a periodic orbit lying outside this homoclinic orbits through $C_{1}$ which intercepts the homoclinic orbit through $B_{1}$, and hence trapped waves will then occur. A typical phase plane is shown in Fig. 8, which is the same case as Fig. 2, now extended to include the relevant orbit (dash-dot curve) from the phase plane for $-L / 2<x<L / 2$. We see that there is a large-amplitude periodic orbit from the origin of the phase plane in the forcing region, which intercepts the homoclinic orbit through $B_{1}$. For $\gamma<\gamma_{c}$ there is only an unbounded orbit from the origin of the phase plane in the forc-

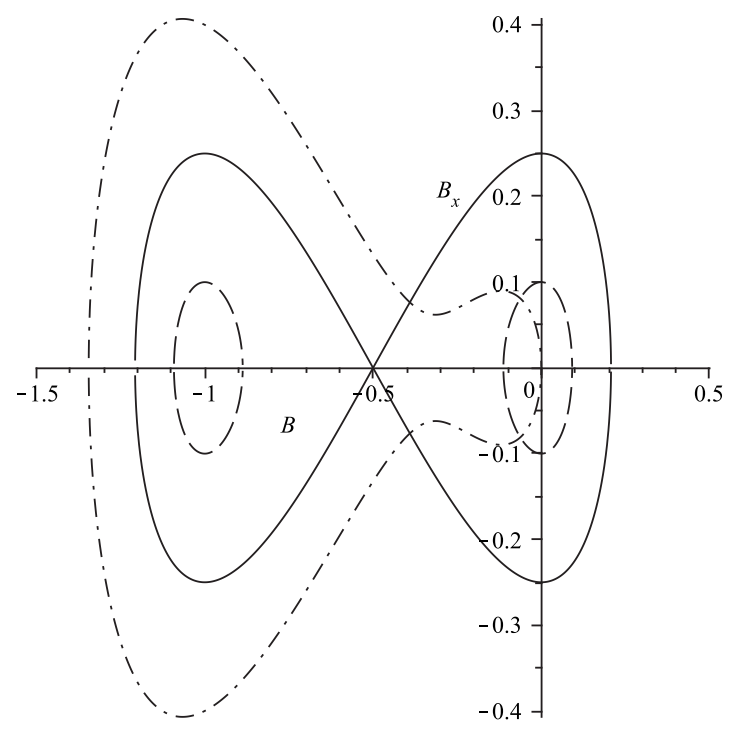

FIG. 8. Phase plane of unforced Eq. (7) for $\Delta=1, q=2$, showing a periodic orbit (dash-dot curve) for the forced equation when $\gamma=-0.08$. 


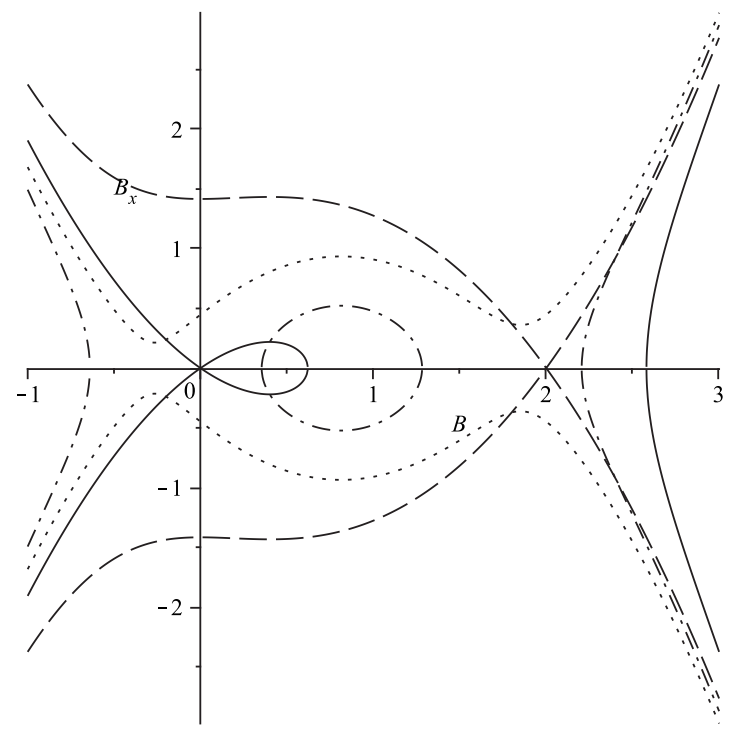

FIG. 9. Phase plane for unforced Eq. (7) for $\Delta=-1, q=-1.25$, showing a periodic orbit (dash-dot curve) for the forced equation when $\gamma=0.5$.

ing region, and this intercepts the homoclinic orbit through $B_{1}$, giving a combined monotonic orbit with $B<0, B_{x}<0$.

\section{C. $\Delta<0$}

As discussed above, the upstream critical point $B=0$ is now a saddle point, and there are two new cases to consider. A typical phase plane for unforced equation (7) is shown in Fig. 9, which is a case (1a) where $q<0,4 \Delta q<9$ when there are two saddle points, $B=0$ and $B=B_{2}$. To obtain a solution when the downstream condition is $B=0$, all that is required is that an orbit (dashdot curve) from the phase plane in the forcing region $-L / 2<x<L / 2$ intercepts the orbit through the origin of the phase plane (solid curve) twice, with the length of the intercept being equal to $L$. Since there is an infinite number of such intercepts, clearly we can expect that there will be ranges of $L$ where at least one solution can be found, which may be periodic as shown for the case in Fig. 9. Similarly, when the downstream condition is that the solution tends to $B_{2}$, that is, case (1a) with $q<0,4 \Delta q<9$, what is required is that an orbit (dot curve) from the phase plane in the forcing region $-L / 2<x<L / 2$ intercepts the orbit through the origin of the phase plane (solid curve) once and then intercepts the orbit through the critical point $B_{2}$ (dash curve) also once, with the length of the intercept being equal to $L$. However, only those orbits on which $x$ increases are allowed, and this corresponds to clockwise in Fig. 9. We see that, in fact, there is no allowed connection.

\section{SLOWLY VARYING APPROXIMATION}

If it is supposed that $f(x)$ is slowly varying, then we assume that $B(x)$ is also slowly varying and seek approximate solutions by omitting $B_{x x}$ in Eq. (7), that is,

$$
\Delta B+3 B^{2}+q B^{3}=-\gamma f(x) .
$$

This is a cubic equation for $B(x)$ given $f(x)$. The situation can be analyzed by arguments similar to the piecewise con- stant case in Sec. III, essentially by examining the existence or otherwise of solutions to the cubic equation for $B$ in Eq. (22). This is the approach used by Grimshaw and Smyth ${ }^{1}$ to find local steady hydraulic solutions for fKdV equation (1) and by Grimshaw et al. ${ }^{10}$ for forced eKDV equation (2) when $q>0$. Note that this slowly varying approximation eliminates the possibility to find waves in the forcing region, as these require the retention of the dispersive term $B_{x x}$ in Eq. (7). But apart from this deficiency, we expect that it will provide useful information supplementing that of Sec. III.

\section{A. Negative forcing: $\gamma>0$}

Suppose first that $\Delta>0, q<0$. Then since we require that $B=0$ is a solution upstream, $x<0$, it follows that there are no solutions which connect to $B_{1}$ downstream. The slowly varying approximation eliminates all waves. Instead there is a connection downstream back to $B=0$ whenever $\gamma$ $<\gamma_{c}$ where $q^{2} \gamma_{c}=2(1-q \Delta / 3)^{3 / 2}+2-q \Delta$. However, this connection is to a center and is an artifact of the slowly varying approximation. A better possibility would be a connection to $B_{2}$, which requires that $\gamma=\gamma_{c}$. This should be an approximation to the parametric map for this case. Next suppose that $\Delta>0, q>0$, when it is readily shown that the only possibility is the connection downstream back to $B=0$, and again this is an artifact of the slowly varying approximation.

If instead $\Delta<0, q<0$, then it is readily shown again that the only possibility is the connection downstream back to $B=0$. This is an allowed saddle point/saddle point connection, and there is no constraint on $\Delta$ or $\gamma$. But if $\Delta<0, q$ $>0$, then there is a connection downstream to $B=0$ when $0<\gamma<\gamma_{c}$, an allowed saddle point/saddle point connection, or a connection downstream to the center at $B_{2}$ when $\gamma=\gamma_{c}$, a parametrically constrained connection. For $\gamma<\gamma_{c}$ there are no solutions. Here $q^{2} \gamma_{c}=2(1-q \Delta / 3)^{3 / 2}+2-q \Delta$.

\section{B. Positive forcing: $\gamma<0$}

Suppose first that $\Delta>0, q<0$. There is a connection downstream back to $B=0$ whenever $\gamma_{c}<\gamma<0$, where now $q^{2} \gamma_{c}=-2(1-q \Delta / 3)^{3 / 2}+2-q \Delta$. However, this connection is to a center and is an artifact of the slowly varying approximation. When $\gamma=\gamma_{c}$ there is an allowed connection to $B_{1}$, and this should be an approximation to the parametric map for this case. The same scenario holds for $\Delta>0, q>0,4 \Delta q$ $<9$. If $\Delta>0, q>0,9<4 \Delta q<12$, then only the connection downstream to $B=0$ when $\gamma_{c}<\gamma<0$ is allowed. If $\Delta<0, q$ $<0, q \Delta>3$, the connection downstream to $B=0$ is allowed for all $\gamma$. But in both these last two cases, the connection is to a center and is as an artifact of the slowly varying approximation.

If instead $\Delta<0, q<0,4 \Delta q<9$, then there is either a connection downstream to $B=0$ when $\gamma_{c}<\gamma<0$, which is an allowed saddle point/saddle point connection, or a connection downstream to $B_{1}$ when $\gamma=\gamma_{c}$, a parametrically constrained saddle point/center connection. If $\Delta<0, q<0,9$ $<4 \Delta q<12$, then only the connection downstream to $B=0$ when $\gamma_{c}<\gamma<0$ is allowed. If $\Delta<0, q<0, q \Delta>3$, the connection downstream to $B=0$ is allowed for all $\gamma$. But if $\Delta$ $<0, q>0$, then there is a connection downstream to $B=0$ 
when $\gamma_{c}<\gamma<0$, an allowed saddle point/saddle point connection, or a connection downstream to the center at $B_{1}$ when $\gamma=\gamma_{c}$, a parametrically constrained connection. For $\gamma<\gamma_{c}$ there are no solutions.

\section{NUMERICAL SOLUTIONS}

Based on the discussion in Sec. II on the upstream and downstream critical points, and on the special cases discussed in Secs. III and IV, we can identify several cases which can be investigated numerically. We will fix $q$ either at a negative value $q=-4$ or at a positive value $q=2$, but will vary $\Delta$ and $\gamma$. In all cases we specify that upstream as $x \rightarrow$ $-\infty, B, B_{x} \rightarrow 0$.

The main case of interest is when $\Delta>0$ and there is a connection between a center $B_{0}=0$ upstream and a saddle point downstream. This will lead to a parametric map $\Delta$ $=\Delta(\gamma)$. Within this case there are several subcases, depending on the relative signs of $q, \gamma$. From Secs. III and IV we expect that downstream the relevant saddle point will usually be $B_{1}$, Eq. (8). For negative forcing $\gamma>0$, we expect to find solutions with trapped waves in the forcing region, and also some solutions without any trapped waves. But for positive forcing $\gamma<0$, we expect to only find solutions with trapped waves when $q>0$. We shall call this class $1 a, b$ depending on whether $\gamma>0,<0$, respectively.

Again choosing $\Delta>0$, there is a possibility of a connection between a center $B_{0}=0$ upstream and a saddle point $B_{2}$ downstream. This case can occur only when $q<0$ and we expect it to occur only for negative forcing $\gamma>0$. We shall call this class 2 .

A new feature for forced extended $\mathrm{KdV}$ equation (2) is the possibility of a connection of a saddle point upstream and a saddle point downstream. This requires $\Delta<0$, so that $B_{0}$ $=0$ is a saddle point. Downstream the allowed saddle point is either $B_{0}=0$ again or $B_{2}$ which occurs only when $q$ $<0,4 \Delta q<9$. The former case is a localized solution in the forcing region, and we expect solutions similar to the examples. ${ }^{19,20}$ However, unlike these examples, there is a possibility that these solutions may contain waves in the forcing region. We shall call this class 3(a). The connection to $B_{2}$ is a new feature not present for fKdV equation (1), although for the case of piecewise constant forcing discussed in Sec. III, there are no such solutions, and also there are no such solutions in the slowly varying approximation, see Sec. IV. We shall call this case 3(b).

As discussed above in Sec. II, we seek solutions such that upstream $B, B_{x} \rightarrow 0, x \rightarrow-\infty$ and downstream $B$ $\rightarrow B_{d}, B_{x} \rightarrow 0$, where $B_{d}$ is the relevant saddle point, either $B_{1}$ or $B_{2}$ as discussed above. For given $q, L$ we vary $\gamma$ and $\Delta$, we can obtain the parametric curve $\Delta=\Delta(\gamma)$. The numerical procedure outlined by Ee and Clarke ${ }^{15}$ was modified and implemented to solve Eq. (7) with the forcing term given by Eq. (6). A summary of the numerical procedure follows, while more details can be found in the referred paper.

- Shoot from the center $(0)$ to a saddle point $\left(B_{d}\right)$ using the Runge-Kutta method of RK(4,5).

- Apply a minimization algorithm to obtain a minimum $\Delta$ so that $B_{d}$ lies within the homoclinic orbit.

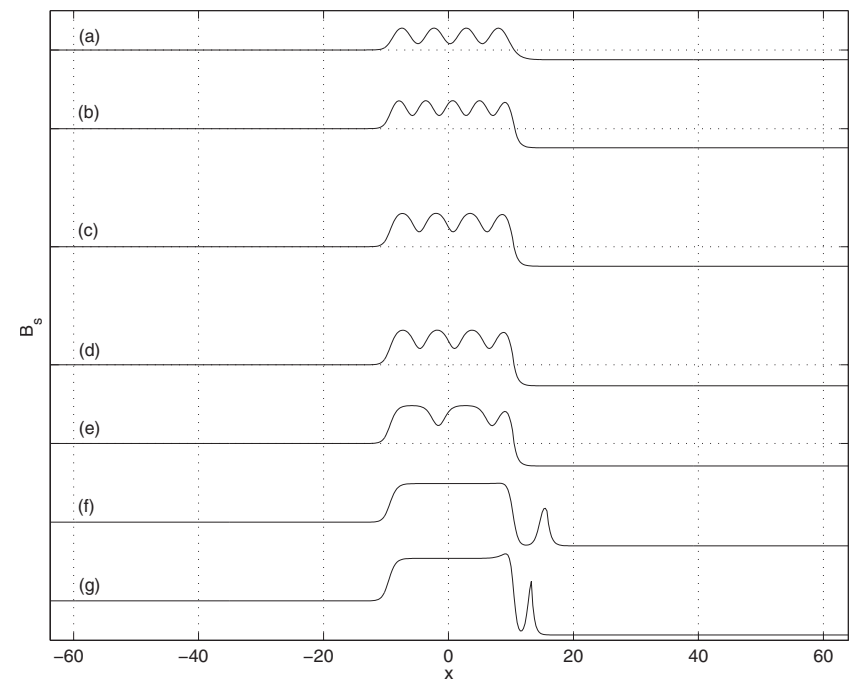

FIG. 10. Plots of steady solutions to Eq. (7) subject to forcing Eq. (6) with $q=-4, L=20$, and the following data values: (a) $\gamma=0.54$ and $\Delta=0.99806$; (b) $\gamma=1.47$ and $\Delta=2.4139$; (c) $\gamma=1.64$ and $\Delta=2.4715$; (d) $\gamma=1.86$ and $\Delta$ $=2.743$; (e) $\gamma=2.1$ and $\Delta=3.0143$; (f) $\gamma=2.3$ and $\Delta=3.252$; (g) $\gamma=4.5$ and $\Delta=5.5939$.

- Insist on the constraints of exponential decay to remove downstream solitary waves appended to the steady dispersive hydraulic solution.

- Employ a branch-following algorithm to obtain the parametric curve $\Delta(\gamma)$.

The branch-following algorithm was initiated from the trivial $(\gamma=0)$ and solitary wave solutions $(\gamma \neq 0)$.

\section{A. Class 1(a): $\Delta>0, \gamma>0$}

Here we first set $q=-4$. In Fig. 10, some plots of the numerical solutions to Eq. (7) subject to forcing (6) are shown. Each plot corresponds to different parts of the parametric map shown in Fig. 11 as indicated. We note three distinct phases. First is the domain $0<\gamma<1.64$ represented by plots (a) and (b) in Fig. 10. This is essentially similar to the $\mathrm{KdV}$ case of Ee et al., ${ }^{14}$ where there are several loops, and as $\gamma$ increases through each loop a new wave crest emerges. Consequently, we did not perform many runs in this region, and hence the loop structure is rather coarsely represented. Compared to the analysis of the piecewise forcing case in Sec. III, this phase corresponds to the regime $\gamma$ $<\gamma_{c 2}$ plotted in Fig. 4.

The second phase is the domain $1.64<\gamma<2.3$ which is marked initially by several rapid oscillations as $\gamma$ increases along the parametric curve, followed by a smoother portion. The first portion is represented by plots (b) and (c) in Fig. 10. At the trough of each oscillation, a trapped wave is lost while at the crest of each oscillation a trapped wave is gained. This portion of the parametric curve we interpret as an adjustment to a transition phase represented by plots (c)-(f) in Fig. 10. In this phase the number of trapped waves decreases and eventually a plateau is formed in the forcing region followed by a single wave. Compared to the analysis of the piecewise forcing case in Sec. III, this phase corresponds to the regime $\gamma_{c 2}<\gamma$ plotted in Fig. 5. The third phase is the domain $\gamma$ 


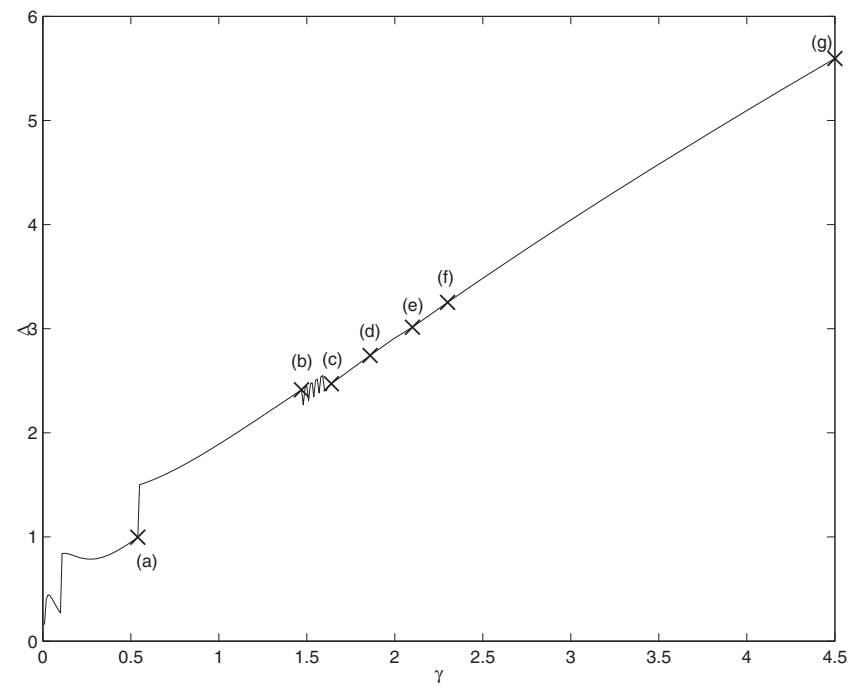

FIG. 11. Parametric map $\Delta(\gamma)$ of family of steady asymmetric solutions to Eq. (7) subject to forcing Eq. (6) using $q=-4$ and $L=20$ and the following data points: (a) $\gamma=0.54$ and $\Delta=0.99806$; (b) $\gamma=1.47$ and $\Delta=2.4139$; (c) $\gamma=1.64$ and $\Delta=2.4715$; (d) $\gamma=1.86$ and $\Delta=2.743$; (e) $\gamma=2.1$ and $\Delta$ $=3.0143$; (f) $\gamma=2.3$ and $\Delta=3.252$; (g) $\gamma=4.5$ and $\Delta=5.5939$.

$>2.3$ and is represented by plots (f) and (g) in Fig. 10. Here only the plateau followed by a single wave is found.

Now, we set $q=2$ and refer to Figs. 12 and 13, which show the typical loop-behavior seen by Ee et al. ${ }^{14}$ in that the loops denote the generation of a trapped wave within the forcing region of the steady solution. The solutions shown in Fig. 12 correspond to the second loop, where $0.05<\gamma$ $<0.18$. As in the study of Ee et al. ${ }^{14}$ the branch number $n$ corresponds to the number of trapped waves $n$ generated. For larger values of $\gamma$, we see that there are gaps between the branches and we shall call this pseudoloop behavior. Note that as $\gamma$ increases the gaps and the loops widen, reflecting that the forcing region is becoming saturated with trapped

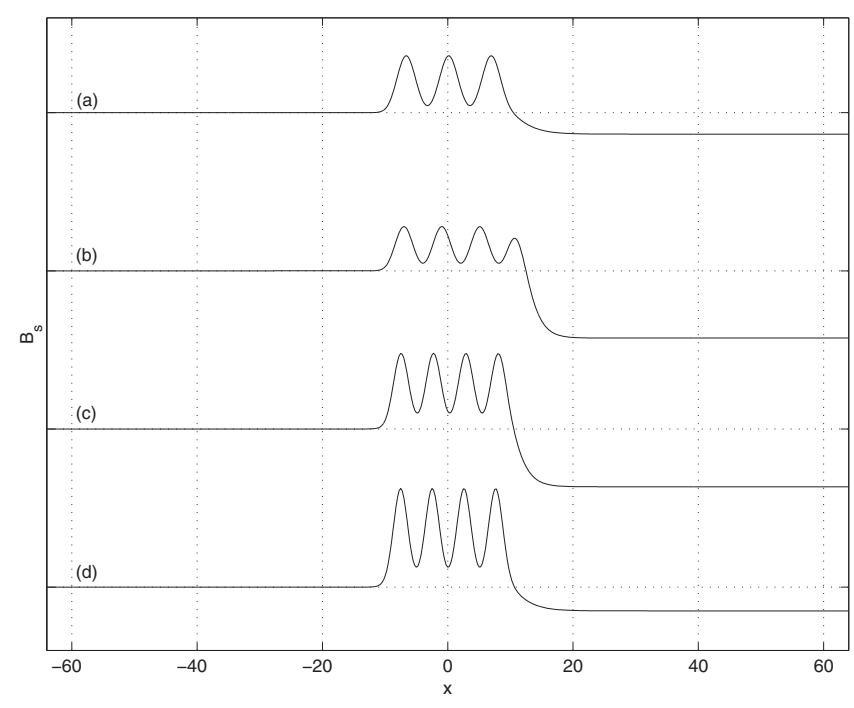

FIG. 12. Plots of steady solutions to Eq. (7) subject to forcing Eq. (6) with $q=2$ and the following data values: (a) $\gamma=0.06$ and $\Delta=0.19443$; (b) $\gamma$ $=0.07$ and $\Delta=0.5427$; (c) $\gamma=0.15$ and $\Delta=0.4812$; (d) $\gamma=0.18$ and $\Delta$ $=0.21385$.
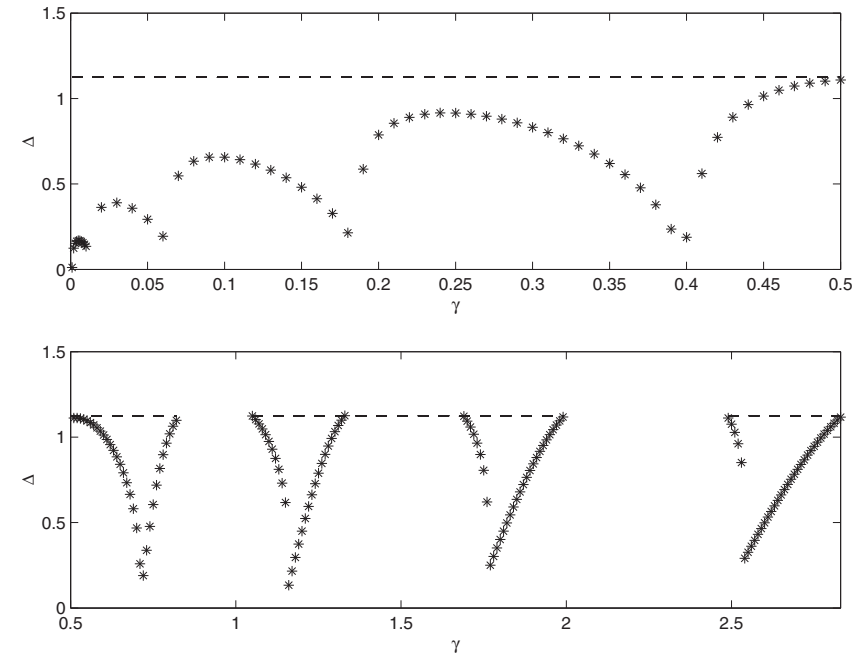

FIG. 13. Parametric map $\Delta(\gamma)$ of family of steady asymmetric solutions to Eq. (7) subject to forcing Eq. (6) using $q=2$. The dotted line above the parametric plot is $\Delta=9 / 4 q$.

waves. The effect intensifies as $L$ increases. Also note that as predicted from the discriminant of Eq. (8), there are no solutions for $\Delta>9 / 4 q$.

\section{B. Class 1(b): $\Delta>0, \gamma<0$}

For $q=-4$, the results are qualitatively similar to those found by Ee et al. ${ }^{14}$ for the $\mathrm{fKdV}$, and, in particular, no trapped waves are found in the forcing region. Some typical solutions and the parametric map are shown in Fig. 14.

For $q=2$ we show some typical solutions in Fig. 15 and the corresponding parametric map in Fig. 16. Note that again the parametric map is truncated above $\Delta=9 / 4 q$ as predicted. Here we see pseudoloops in that for small values of $|\gamma|$ branches 1 and 2 overlap, but are not connected, while for larger values of $|\gamma|$, there are gaps between the branches. The
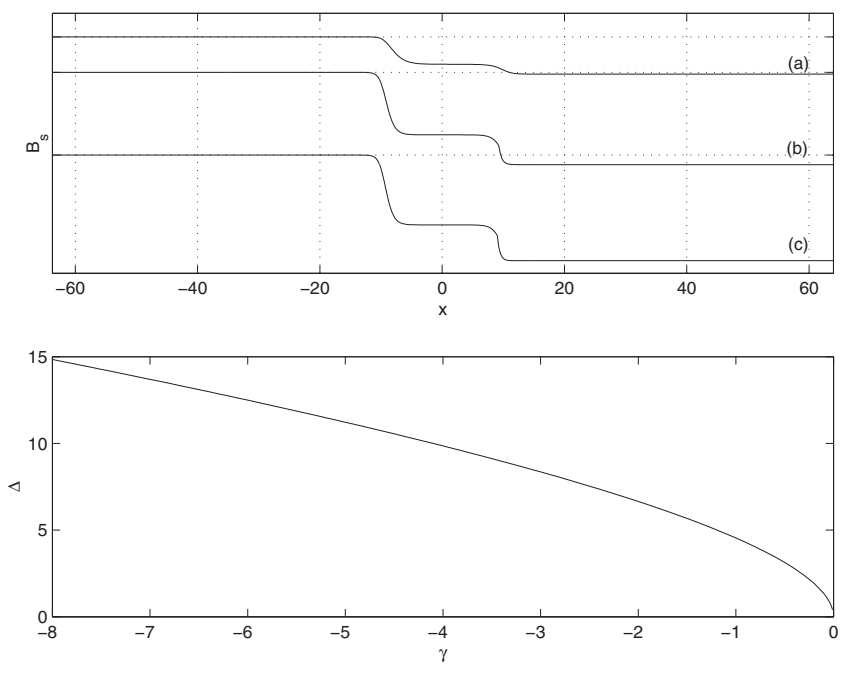

FIG. 14. Top panel: Plots of steady solutions to Eq. (7) subject to forcing Eq. (6) using $q=-4, L=20$, and the following data values: (a) $\gamma=-0.1$ and $\Delta=1.3421$; (b) $\gamma=-1.1$ and $\Delta=4.7857$; (c) $\gamma=-1.6$ and $\Delta=5.8769$. Bottom panel: Parametric map $\Delta(\gamma)$ of family of steady solutions to Eq. (7) subject to forcing Eq. (6) with $q=-4$. 


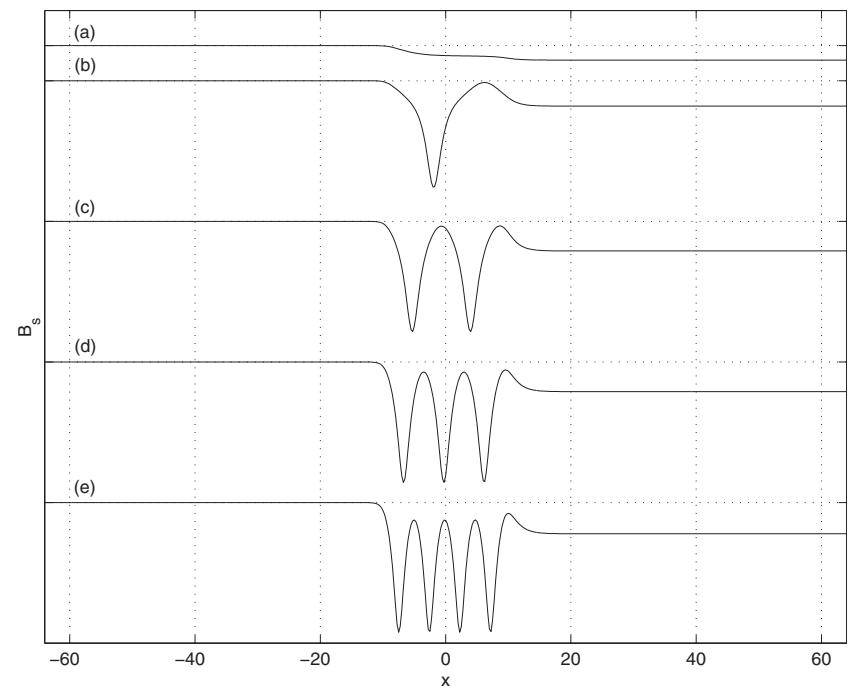

FIG. 15. Plots of solutions to Eq. (7) subject to forcing Eq. (6) using $q=2$, $L=20$, and the following data values: (a) $\gamma=-0.02$ and $\Delta=0.53233$; (b) $\gamma$ $=-0.07$ and $\Delta=0.82075$; (c) $\gamma=-0.19$ and $\Delta=0.9083$; (d) $\gamma=-0.39$ and $\Delta=0.90984$; (e) $\gamma=-0.69$ and $\Delta=0.9376$. The dotted line above the parametric plot defines the upper limit of the parametric map $\Delta=9 / 4 q$.

solutions shown in Fig. 15 correspond to the branches 1-5 for $-0.8<\gamma<0$. Again, the branch number $n$ corresponds to the number of trapped waves $n$ generated. However, note that there are initial transitions between branches 1 and 2 . Also, the smallest $\Delta$ value associated with each branch is very small for the first two branches of the parametric map, see the top panel of Fig. 16, whereas it is quite large for the remaining branches. As with Fig. 13, the gap between the branches also widens as $|\gamma|$ increases.

\section{Class 2: $\Delta>0, \gamma>0, q<0$}

Although the classification described above suggests that there may be a connection to a saddle point $B_{2}$ downstream, we were unable to find numerically any such solutions for this case.
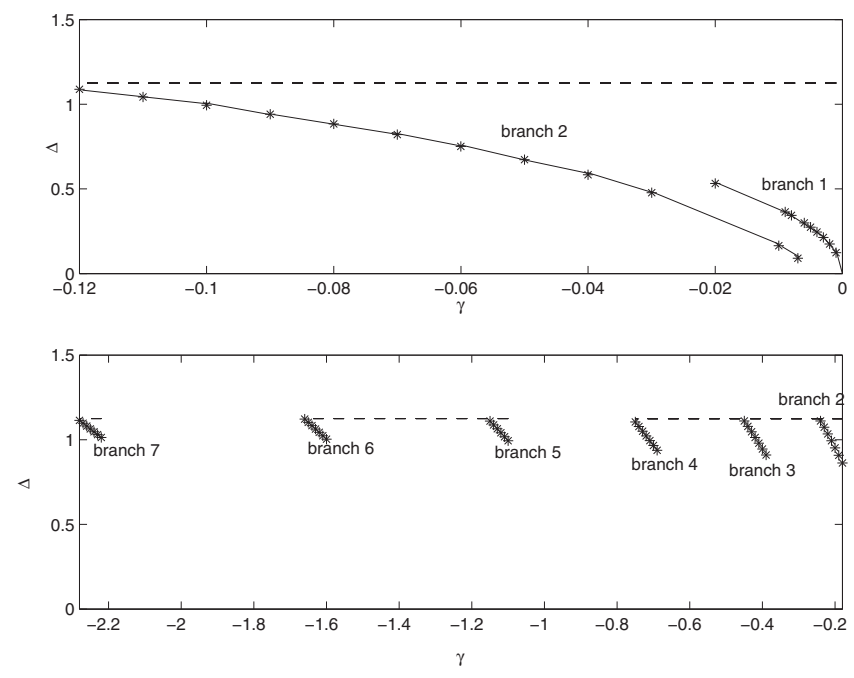

FIG. 16. Parametric map $\Delta(\gamma)$ of family of solutions to Eq. (7) subject to forcing Eq. (6) using $q=2$ and $L=20$. The dotted line above the parametric plot defines the upper limit of the parametric map $\Delta=9 / 4 q$. The lines featured in the top panel denote the two branches of steady solutions for $\gamma$ $\in[-0.12,0]$.

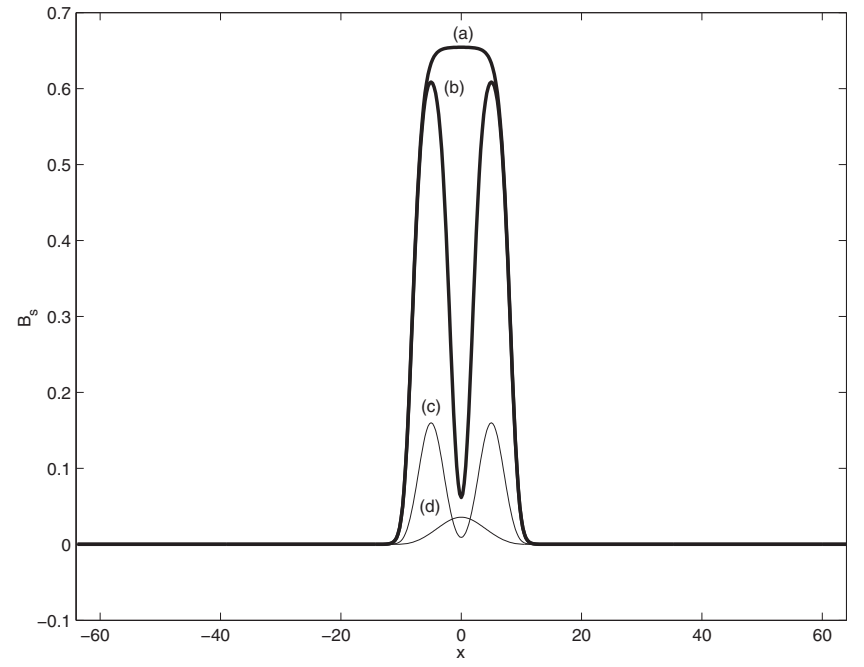

FIG. 17. Plots of solutions to Eq. (7) subject to forcing Eq. (6) using $q=-4$, $L=20, \Delta=-0.001$, and the following data values: (a) $\gamma=0.16288$ (larger amplitude solution resembling a table-top soliton); (b) $\gamma=0.16055$ (larger amplitude solution with two trapped waves); (c) $\gamma=0.022734$ (smaller amplitude solution with two trapped waves); (d) $\gamma=0.0012305$ (smaller amplitude solution with one trapped wave).

\section{Class 3(a): $\Delta<0, \gamma>0, q<0$}

In Fig. 17 we observe several types of steady symmetric solutions that can occur: standard solitary wave, pseudobreather, and table-top solitary wave solutions. We observe that as $\gamma$ increases, the magnitude of the standard solitary wave increases and undergoes a transformation to a table-top solitary wave solution. The pseudobreathers are a result of the nature of the forcing which allows trapped waves to be formed in the forcing region. We also observe that as two branches approach each other but never meet, the table-top solitary wave solution encloses the pseudobreathers.

\section{E. Class 3(b): $\Delta<0, \gamma>0, q<0$}

Although the classification described above suggests that there may be a connection between the saddle points $B_{0}=0$ upstream to a saddle point $B_{2}$ downstream, we were unable to find numerically any such solutions for this case.

\section{CONCLUSION}

In this paper, we have investigated the transcritical flow of a stratified fluid over an obstacle, in the framework of the forced extended Korteweg-de Vries equation. Our concern is solely with the steady hydraulic solutions which may form over the obstacle, which can be characterized as either a hole or a hill depending on its polarity relative to the quadratic nonlinear term in governing Eq. (7). Our main finding is that as long as the obstacle is wide enough, trapped waves are formed in the forcing region, and this can occur for both holes and hills.

This work extends the analogous study by Ee et al. ${ }^{14}$ for the fKdV equation, and so a central issue is the role played by cubic nonlinearity in the formation of trapped waves. From the underlying theory presented in Sec. II and for the special case of piecewise constant forcing discussed in 
Sec. III, the main effect of the cubic nonlinearity is to increase the number of available critical points from two for the $\mathrm{KdV}$ case to three for the $\mathrm{KdV}$ case. When the extra critical point is "passive," i.e., it plays no essential role in the phase plane analysis, then the present results are generally qualitatively similar to those reported by Ee et al. ${ }^{14}$ for the $\mathrm{KdV}$ case. An example of this for class 1 (a) when $\Delta>0, \gamma$ $>0$ with $q<0$ is shown in Fig. 4 where the phase plane is similar to the corresponding phase plane for the KdV case. But then in Fig. 5, the phase plane has a structure not allowed for the KdV case. This essential difference is replicated in the numerical results of Figs. 10 and 11, where plots (a) and (b) are qualitatively similar to the analogous $\mathrm{KdV}$ case, but plots (c)-(g) can only arise in the eKdV case. Again for class 1(a) but with $q>0$, the phase plane is that of Fig. 6 which is similar to the $\mathrm{KdV}$ case, and the numerical results of Fig. 12 are also similar to the corresponding KdV case; but here the parametric map shown in Fig. 13 displays some significant differences as $\gamma$ increases with the generation of pseudoloop behavior.

When $\Delta>0, \gamma<0$, class $1(\mathrm{~b})$, typical phase planes are shown in Figs. 7 and 8 for $q<0, q>0$, respectively, and the corresponding numerical results are shown in Figs. 14 and 15. Here for the $\mathrm{KdV}$ case, there are no trapped waves formed, and this is also seen here when $q<0$. But for $q$ $>0$, solutions with trapped waves can be found, a feature which is absent for the $\mathrm{KdV}$ case and hence can arise only in the eKdV case. Class 3(a) when $\Delta<0$ corresponds to localized steady solitary waves trapped by the forcing region. This case was not explicitly discussed by Ee et al., ${ }^{14}$ but it is well known that such solutions exist. That is also confirmed here for the eKdV case, which when $q<0$ exhibits a wide range of solution types, see Fig. 17.

\section{ACKNOWLEDGMENTS}

Partial financial support has been provided by the Research Grants Council Contract No. HKU 7120/08E and the University of Hong Kong Seed Funding Program for Basic Research Grant No. 200911159076.

${ }^{1}$ R. H. J. Grimshaw and N. Smyth, "Resonant flow of stratified fluid over topography," J. Fluid Mech. 169, 429 (1986).
${ }^{2}$ S. R. Clarke and R. H. J. Grimshaw, "Resonantly generated internal waves in a contraction," J. Fluid Mech. 274, 139 (1994).

${ }^{3}$ R. Grimshaw, "Transcritical flow past an obstacle," ANZIAM J. (to be published).

${ }^{4}$ R. Grimshaw and E. Pelinovsky, "Interaction of a solitary wave with an external force in the extended Korteweg-de Vries equation," Int. J. Bifurcat. Chaos Appl. Sci. Eng. 12, 2409 (2002).

${ }^{5}$ P. G. Baines, Topographic Effects in Stratified Flows (Cambridge University Press, Cambridge, 1998).

${ }^{6}$ A. Slyunyaev and E. Pelinovsky, "Dynamics of large-amplitude solitons," Sov. Phys. JETP 89, 173 (1999).

${ }^{7}$ A. Slyunyaev, "Dynamics of localized waves with large amplitude in a weakly dispersive medium with a quadratic and positive cubic nonlinearity," Sov. Phys. JETP 92, 529 (2001).

${ }^{8}$ R. Grimshaw, D. Pelinovsky, E. Pelinovsky, and A. Slunyaev, "The generation of large-amplitude solitons from an initial disturbance in the extended Korteweg-de Vries equation," Chaos 12, 1070 (2002).

${ }^{9}$ J. W. Choi, S. M. Sun, and M. C. Shen, "Internal capillary-gravity waves of a two-layer fluid with free surface over an obstruction - Forced extended KdV equation," Phys. Fluids 8, 397 (1996).

${ }^{10}$ R. H. J. Grimshaw, K. H. Chan, and K. W. Chow, "Transcritical flow of a stratified fluid: The forced extended Korteweg-de Vries model," Phys. Fluids 14, 755 (2002).

${ }^{11}$ R. H. J. Grimshaw, D.-H. Zhang, and K. W. Chow, "Generation of solitary waves by transcritical flow over a step," J. Fluid Mech. 587, 235 (2007).

${ }^{12}$ D.-H. Zhang and A. T. Chwang, "Generation of solitary waves by forward- and backward-step bottom forcing," J. Fluid Mech. 432, 341 (2001).

${ }^{13}$ R. H. J. Grimshaw, D.-H. Zhang, and K. W. Chow, "Transcritical Flow Over a Hole," Stud. Appl. Math. 122, 235 (2009).

${ }^{14}$ B. K. Ee, R. H. J. Grimshaw, K. W. Chow, and D.-H. Zhang, "Steady transcritical flow over a hole: Parametric map of solutions of the forced Korteweg-de Vries equation," Phys. Fluids 22, 056602 (2010).

${ }^{15}$ B. K. Ee and S. R. Clarke, "Weakly dispersive hydraulic flows in a contraction - parametric solutions and linear stability analysis," Phys. Fluids 19, 056601 (2007).

${ }^{16}$ R. Grimshaw, M. Maleewong, and J. Asavanant, "Stability of gravitycapillary waves generated by a moving pressure disturbance in water of finite depth," Phys. Fluids 21, 082101 (2009).

${ }^{17}$ F. Dias and J. M. Vanden-Broeck, "Generalized critical free surface flows," J. Eng. Math. 42, 291 (2002).

${ }^{18}$ F. Dias and J. M. Vanden-Broeck, "Steady two-layer flows over an obstacle," Philos. Trans. R. Soc. London, Ser. A 360, 2137 (2002).

${ }^{19}$ F. Dias and J. M. Vanden-Broeck, "Trapped waves between submerged obstacles,” J. Fluid Mech. 509, 93 (1999).

${ }^{20}$ B. J. Binder, J.-M. Vanden-Broeck, and F. Dias, "Forced solitary waves and fronts past submerged obstacles," Chaos 15, 037106 (2005).

${ }^{21}$ B. J. Binder, F. Dias, and J.-M. Vanden-Broeck, "Steady free-surface flow past an uneven channel bottom," Theor. Comput. Fluid Dyn. 20, 125 (2006).

${ }^{22}$ F. Dias and J. M. Vanden-Broeck, "Two-layer hydraulic falls over an obstacle,” Eur. J. Mech. B/Fluids 23, 879 (2004). 